\title{
Influence of Particle Shape on the Macroscopic and Mesolevel Mechanical Properties of Slip Zone Soil Based on 3D Scanning and 3D DEM
}

\author{
Zechuang Li $(\mathbb{D}$ and Zhibin Liu ii \\ School of Civil Engineering, Northeast Forestry University, Harbin 150040, China \\ Correspondence should be addressed to Zhibin Liu; lzbnefu@163.com
}

Received 8 July 2021; Revised 25 September 2021; Accepted 27 September 2021; Published 9 October 2021

Academic Editor: Hao Yi

Copyright ( 92021 Zechuang Li and Zhibin Liu. This is an open access article distributed under the Creative Commons Attribution License, which permits unrestricted use, distribution, and reproduction in any medium, provided the original work is properly cited.

\begin{abstract}
The macroscopic and mesolevel mechanical mechanisms of slip zone soil are a crucial subject for the research of landslide deformation evolution and slope control, but the effects of the shape and psephicity of coarse particles in a slip zone soil on the mechanical properties of the slip soil zone still need to be explored. Discrete element method (DEM) can effectively monitor and track the mesolevel mechanical parameters of geotechnical materials, such as displacement vector field, contact force chain, and particle coordination number. The rock blocks in the medium-sized shear test undergo a sophisticated process by 3D scanning technology, and a database of the blocks is established and accurately modeled by combining 3D DEM to simulate the indoor medium-sized shear test for numerical investigation in line with the test conditions. The numerical simulation results demonstrate that the psephicity and particle shape of the rock blocks significantly affect the dilatancy and mesolevel mechanical parameters of the slip zone soil specimens. In addition, the numerical models featured by poorer psephicity and more irregular particle shape display more evident dilatancy, larger particle coordination numbers, as well as better contact density inside the model. Some references for the study of the macroscopic and mesolevel mechanical mechanisms of slip zone soil are provided.
\end{abstract}

\section{Introduction}

The physical and mechanical properties of slip zone soil, which acts as a critical component of the slip zone of landslides, are closely related with the formation and development of landslides. The study of the macroscopic and mesolevel mechanical mechanisms of slip zone soil is of remarkable importance to the research of the deformation and damage mechanisms, prevention, and management of landslides. The composition and formation process of slip zone soil under the influence of geological conditions are complex. Strength-related characteristics, which are the focus of research on slip zone soil, are an important part of the analysis of landslide stability [1]. A large number of researches on slip zone soil have been carried out through various methods, but most of the research objects are finegrained slip zone soil [2-5]. The existence of coarse particles, such as gravel, in slip zone soil makes the basic properties greatly different from that of the general slip zone soil. The presence of gravels and the content of coarse grains have an important influence on the physical and mechanical properties of slip zone soil $[6,7]$.

Field and laboratory tests are important means to study the macroscopic mechanical strength and deformation characteristics of sliding-zone soil. Chen et al. [8] studied the strength characteristics of coarse-grained sliding-zone soil through field and laboratory shear tests and pointed out that this soil is different from general clay and sandy soil in terms of shear properties. Liu et al. $[9,10]$ studied the influence of coarse grain content and microstructure on the strength characteristics of the slip zone soil of an ancient landslide through indoor ring shear test. The study showed that sandbearing slip zone soil has different strength characteristics compared with general clay soil and has no obvious strain 
softening phenomenon; the coarse grain content has an obvious influence on postpeak strength. Li et al. [11] investigated the effects of different shear rates on the mechanical properties of slip zone soil containing coarse grains in the Three Gorges Reservoir area by large-scale ring shear tests. Zou et al. [12] studied the shear mechanical properties of gravelly slip zone soil in the Three Gorges Reservoir area through in situ shear test and found that the presence of gravel improves the shear strength of slip zone soil. Ren et al. [13] pointed out that gravel is the internal factor that causes residual strength change in slip zone soil through direct shear test, and gravel content determines the number of contact surfaces on shear surface. Wu et al. [14] found that gravel content and gravel grade have important influences on the internal friction angle and cohesion of gravelly slip zone soil through direct shear test. Wen et al. [15] found that the presence of gravel significantly affects the creep properties of slip zone soil based on shear creep tests. Ren et al. [16] studied the strength characteristics of pebbly slip zone soil through medium shear test and pointed out that the residual internal friction angle of pebbly slip zone soil is different from that of general slip zone soil, and its value is controlled by gravel and clay contents. Moreover, the presence of gravel can change the roughness of a shear surface and increase residual strength. According to these studies, the existence of coarse particles, such as gravel and block stone, remarkably affects the mechanical and deformation characteristics of slip zone soil.

The macrolevel mechanical properties of slip zone soil are macroscopic manifestations of the mesolevel mechanics between slip zone soil particles. Numerical simulation method can be complemented with macroscopic tests to look into the macroscopic mechanical deformation and strength properties of slip zone soil in a multidimensional manner. Jiang et al. [17] investigated the macroscopic and mesolevel mechanical strength properties and deformation and damage mechanisms of slip zone soil using a combination of CT scanning and triaxial numerical tests. Soil structure can be seen as a system of spatial structure formed by skeletal units, such as single particles, particle clusters, or clods. Soils own complex engineering mechanical properties. Conventional indoor tests can be used to monitor deformation damage on the specimen surface but not the deformation damage inside the specimen and the contact changes between particles. Thus, the influence of mesolevel mechanical parameters, such as interparticle friction angle, on geotechnical properties are difficult to investigate through indoor tests because of the limitations of test methods and test apparatus [18]. The study of such complex engineering properties of soils can be set out from mesolevel morphological pathways. The discrete element method (DEM) can numerically simulate the engineering properties of soils from the mesolevel perspective, observe the mesolevel constitutive characteristics of particles and their changes in real time, and analyze the macroscopic mechanical behaviors of geotechnical materials through the study of the mesolevel parameters of particle structure [19]. Particle discrete elements have been an indispensable means to study the mesolevel mechanics of geotechnical materials, and various scholars at home and abroad have carried out an array of research for geotechnical materials through the agency of the discrete unit method to unveil the intrinsic mechanisms of shear strength and deformation damage of geotechnical materials from the mesolevel point of view [20-27]. However, the literature on discrete element research that targets slip zone soils is still lacking. Previous studies used 3D discrete element to implement numerical simulation research by employing regular spheres or irregular clusters of particles combined with each other [28-31], which fails to fully consider the real shape of particles that differ greatly from rock blocks found in real geotechnical materials in terms of surface roughness and irregularity of shape. Previous studies have revealed that particle shape has an important influence on the macrolevel and mesolevel mechanical properties of geotechnical bodies $[32,33]$. Xu et al. [34] pointed out that the morphology of rock blocks is an important influencing factor for the macrolevel and mesolevel mechanical properties and mesolevel structure of soil-rock mixtures. Jin et al. [35] showed that the shape of blocks affects the mechanical behaviors of soil-rock mixtures, and the degree of influence is closely correlated with rock content. Wang et al. [36] pointed out that the shape of particles from disintegrated rocks can remarkably affect the overall shear performance of the aggregate through the discrete element simulation of the rock aggregate, and the internal friction angle and cohesion of the aggregate increase with the rise of the angularity index. Zhao et al. [37] studied the effect of particle prism angle on direct shear test using DEM and pointed out that the size of particle angularity notably affects the dilatancy and shear strength of the particle system. In summary, particle morphology has an important influence on the mechanical characteristics of geotechnical materials. In this study, the 3D model of real blocks from medium-sized shear tests was obtained through $3 \mathrm{D}$ scanning technique and then imported into 3D DEM to generate the mesolevel structure model. The effect of two different shapes of coarse particles on the macroscopic and mesolevel mechanical strength and deformation damage mechanisms of the slip zone soil was investigated in combination with medium-sized shear test in the laboratory.

\section{Introduction to 3D DEM and 3D Scanning of the Blocks for Test}

2.1. Introduction to $3 D$ DEM. The $3 \mathrm{D}$ discrete element numerical simulation method deployed in this study is Particle Flow Code in 3 Dimensions (PFC3D). Particle Flow Code (PFC) is a mesolevel mechanical analysis software based on the general DEM framework, which consists of a computational engine and a graphical user interface. The particle DEM is the most ideal of the DEM, which treats the discrete body as an aggregate of finite discrete particle units and thus idealizes the materials as a group of particles that are independent of each other, in contact, and interacting with each other. DEM applies the force-displacement law and Newton's law of motion alternately throughout the calculation cycle, updates the contact force in the contact part by the force-displacement law, and updates the particle- 
particle and particle-boundary positions through the law of motion to reach a new equilibrium [18]. Previous studies have shown that numerical tests carried out by numerical models based on the mesolevel parameters of 3D discrete elements can better reproduce the shear properties, macrolevel and mesolevel mechanical properties, strength characteristics, and damage modes of geotechnical media, such as soil-rock mixtures $[35,38,39]$.

2.2. 3D Scanning of the Blocks for Test. 3D modeling of real blocks for testing, which are of close approximation to those used in medium-sized shear test (two types of blocks with notable differences in psephicity as shown in Figure 1(a), was performed through $3 \mathrm{D}$ scanning technology to achieve a more refined simulation in the study. The 3D scanning equipment used is the TEXU-SCAN3D instrument, a 3D optical surface scanning system that is widely used in the reverse design of 3D complex curved surfaces and 3D fullsize inspection. Specifically, the process of rock scanning began by $3 \mathrm{D}$ scanning measurement using a raster projection device to project multiple multifrequency raster to the rocks to be scanned. Two cameras were placed in a certain angle to collect the corresponding images in sync, and then, decoding and phase calculation were performed on the images. Next, stereo matching technology and triangulation principle were applied to calculate the $3 \mathrm{D}$ coordinates of the pixels of the scanned rocks in the common viewing area of the two cameras to obtain a file of the 3D scanning results in STL format. Finally, the file was imported into the Geomagic Studio software for processing to obtain the dimensional parameters of the scanned rocks, such as the surface area and volume.

Twelve stones were scanned in this study, with six stones for each shape or roundness. These stones met the shape description of one kind of stone with great roundness. The white blocks had better psephicity and thus named subround blocks, and the brown blocks were poorer psephicity and termed as angular blocks. Besides, all the scanned blocks were marked for easier distinction. Numbers 1-6 are the subround blocks, and numbers 7-12 are the angular ones. The surface area and volume of each block were calculated as shown in Table 1, and the results of the 3D scanning and modeling are shown in Figure 1(b).

\section{PFC3D Shear Test Simulation}

3.1. Introduction to the Medium-Sized Shear Test. This discrete element numerical simulation was based on the data of indoor medium-sized shear test. The specimen size of the medium-sized shear box is $200 \mathrm{~mm} \times 200 \mathrm{~mm} \times 200 \mathrm{~mm}$. The direct shear box was independently designed and modified. The whole shearing process can be photographed and recorded by single-lens reflex cameras from the front and back of the box. The medium-sized direct shear instrument used in the experiment is shown in Figure 2(a). The research object was the slip zone soil collected from a large landslip zone in Fushun City, Liaoning Province, China. The soil contains a huge number of coarse particles. Medium- sized shear test was utilized to uncover the effect of different contents of coarse particles on the mechanical properties of slip zone soil. The contents of coarse particles were $40 \%$, $50 \%, 60 \%, 70 \%$, and $80 \%$. Experimental research on the impact of the contents of coarse particles in slip zone soil on its mechanical properties will be introduced elaborately in other articles. With reference to previous studies, $2 \mathrm{~mm}$ was selected as the threshold for the boundary between soils and rocks in this study, and three different ranges for coarse particles are classified, namely, 2-5, 5-10, and 10-20 mm. Figure 2 shows the specimen of slip zone soil with a coarse grain content of $50 \%$. Figures 2 (b) and 2(c) show the images of the specimen before and after shearing. For easier observation, the surfaces of certain coarse particles were marked with a cross mark. A comparison of the images before and after medium-sized shear test clearly presents that the coarse particles in the specimen had obvious signs of flipping and drift, and the coarse particles (circled by red lines) in the shearing area at the center exhibited more evident changes.

\subsection{Creation of 3D Discrete Element Numerical Model}

\subsubsection{Generation of the Shear Box for Numerical Test.} The numerical model was created based on the geotechnical materials of the medium-sized shear test, and the size of the numerical model was consistent with the specimen size, which are all $200 \mathrm{~mm} \times 200 \mathrm{~mm} \times 200 \mathrm{~mm}$ cubes. In the numerical investigation, as many as 12 shear walls were used to simulate the shear box, the upper and lower shear boxes have five walls each, and a baffle wall with a length of $60 \mathrm{~mm}$ was placed in the middle of each of the two sides of the shear box. Each wall in PFC3D was composed of two triangular facets to prevent particles from escaping during the shearing process. The shear box used in the numerical simulation is shown in Figure 3. The shearing process was performed under the following conditions: the upper box remained stationary, the left and right walls of the lower box had the same shearing speed, and the left baffle wall was given the same speed to prevent particles from running away during the shearing process. Before starting the shearing, the numerical model was subjected to initial normal stress through the servo program, and then, the servo control was used to keep the normal pressure of the bottom and top walls constant during the shearing process.

3.2.2. Generation of Complex Rock Particle Clumps. In PFC3D, clumps of irregular particles were generated by small regular spheres. Under the same conditions, the effect of the generated particle clumps will be closer to the real blocks, and the simulation effect will be better when more small spheres fill in the particle clump; however, the calculation efficiency will also drop notably. Therefore, maintaining the balance between the number of spheres filled in the particle clump and the efficiency of the discrete element simulation is necessary. In this study, through repeated attempts to explore the number of different spheres in particle clumps, we can finally simulate complex rock 


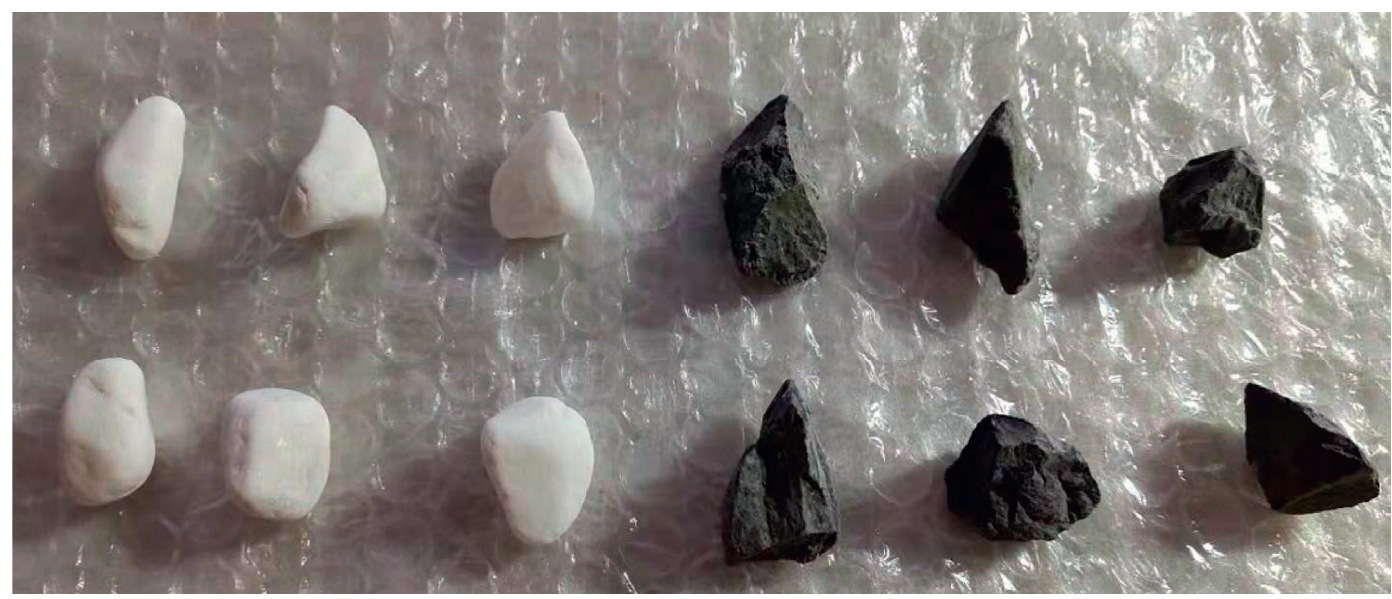

(a)

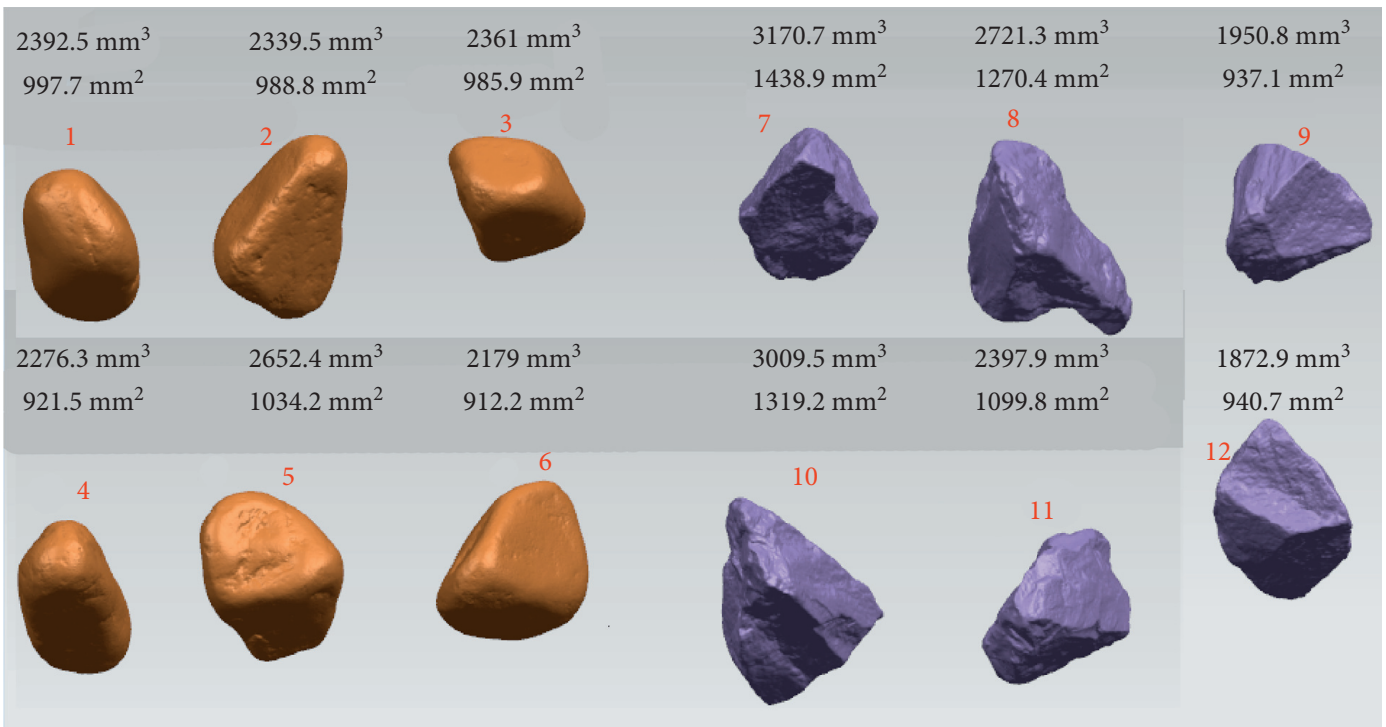

(b)

Figure 1: The subcircular and angular test stones and their 3D scanning images. (a) Test stone. (b) The test stones were scanned.

TABLE 1: Specific parameters of 3D scanned stones.

\begin{tabular}{|c|c|c|c|c|c|c|c|c|c|c|c|c|}
\hline \multirow{2}{*}{ Stone parameters } & \multicolumn{12}{|c|}{ Number } \\
\hline & 1 & 2 & 3 & 4 & 5 & 6 & 7 & 8 & 9 & 10 & 11 & 12 \\
\hline Volt & 392.5 & 2339.5 & 23610 & 2276.3 & 2652.4 & 2179.0 & 3170.7 & 2721.3 & 1950.8 & 3009.5 & 2397.9 & 1872.9 \\
\hline Superficial area $/\left(\mathrm{mm}^{2}\right)$ & 997.7 & 988.8 & 985.9 & 921.5 & 1034.2 & 912.2 & 1438.9 & 1270.4 & 937.1 & 1319.2 & 1099.8 & 940.7 \\
\hline
\end{tabular}

particle clumps with satisfactory model calculation efficiency and accuracy.

The radius of soil particles was kept between 3 and $4 \mathrm{~mm}$, which was represented by a regular sphere ball generated by a random generation algorithm and by specifying the porosity of the specimen space. The irregular coarse particles were imported into PFC3D after scanning the real rocks in all directions based on 3D scanning technology. The coarse particles in the numerical specimen were created using the clump template generation method in PFC3D by importing the $3 \mathrm{D}$ scanned test rocks in STL format through the instruction of geometry import to create the clump template, and then the filling process was performed via the keyword pebblepack, which involves four key parameters: distance controls the degree of roundness of the template within the range of $0-180$, ratio is the radius of the minimum and maximum particles filled in the template and ranges between 0 and 1 , radfactor indicates the proportion of particles within the template whose radius can exceed the boundary, and refinenum indicates the number of optimization attempts to set the grid. The specific parameters used in this study were a distance of 150.0 , a ratio of 0.3 , a radfactor of 1.05 , and a refinenum of 10,000 . As many as three clump templates, named $s_{1}, s_{2}$, and $s_{3}$, were created according to the above method, and the coarse particles in the model were generated by the clump distribute command according to the 


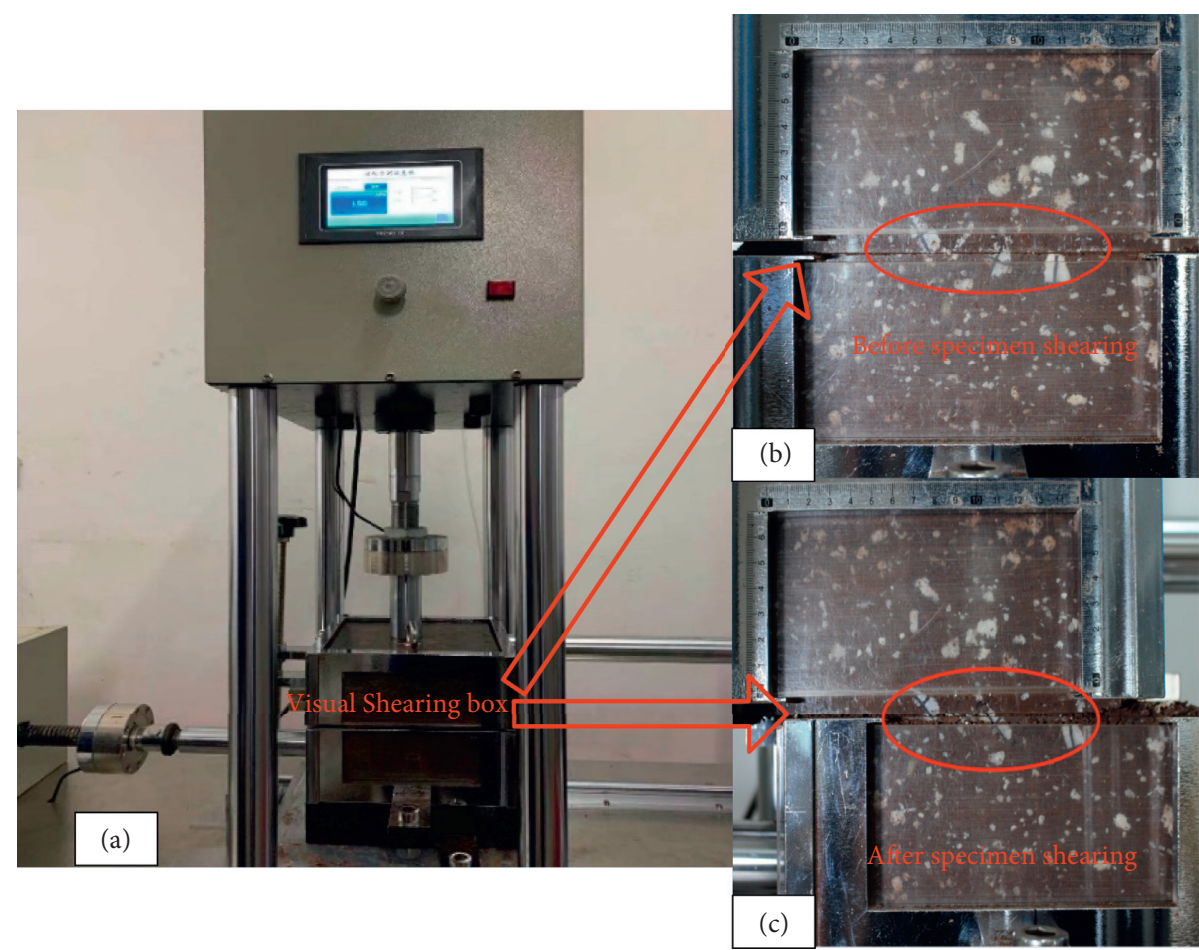

FIGURE 2: Comparison before and after shear test of medium direct shear apparatus and sliding-zone soil samples with $50 \%$ coarse grain content.

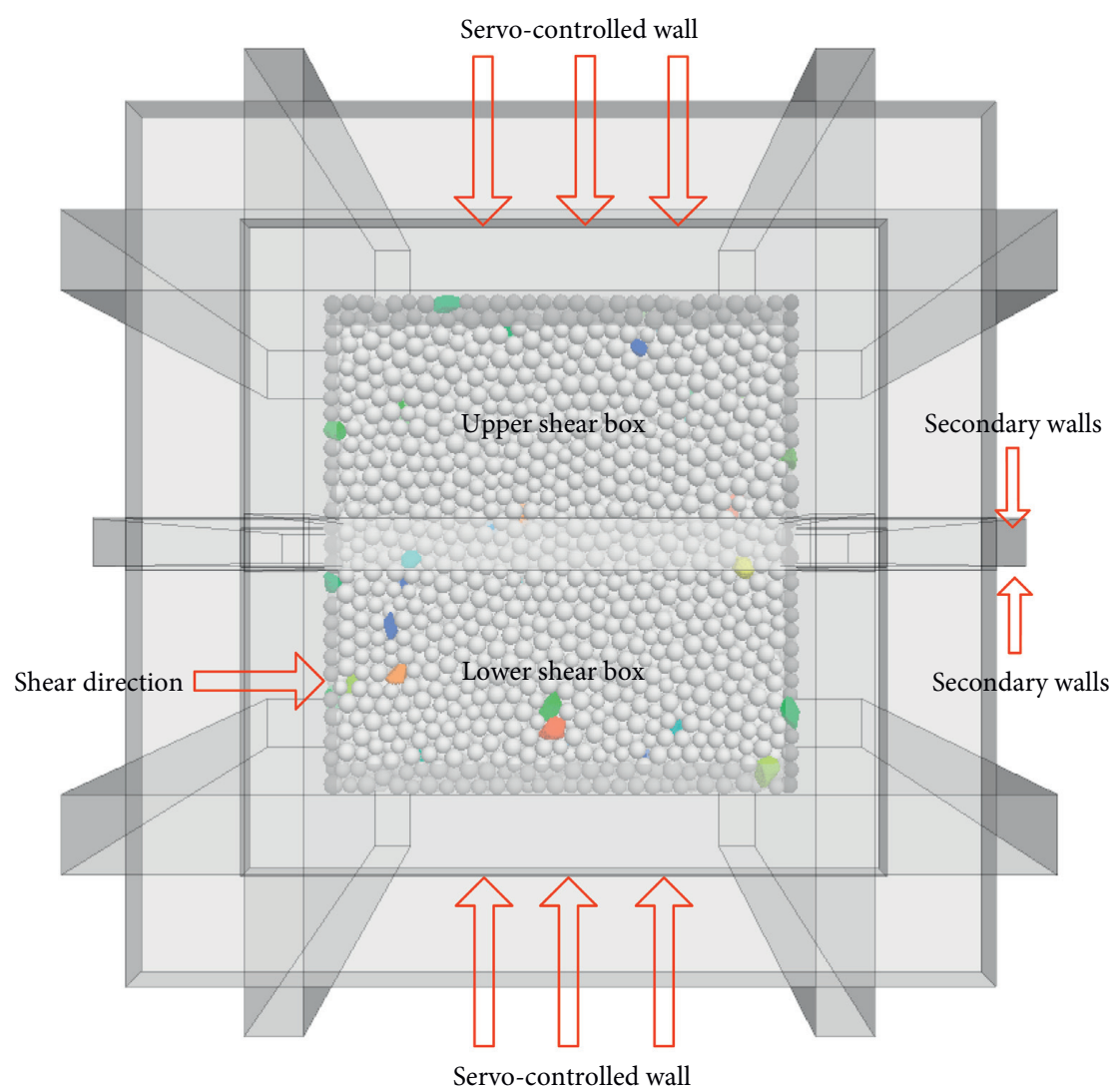

Figure 3: Numerical test shear box. 
Gaussian distribution. The shape of the coarse particles was controlled by the templates, the volume ratios of $s_{1}, s_{2}$, and $s_{3}$ were $0.4,0.3$, and 0.3 , respectively (the sum of the template volume ratio must be 1 ). The minimum and maximum sizes of the coarse particles were 6 and $12 \mathrm{~mm}$, respectively. The generation process of the coarse-fine particle mixture model is shown in Figure 4.

3.2.3. Selection of Numerical Test Contact Model. The selection of the mesolevel contact model between particles in the discrete element numerical simulation is very important. The interparticle contact model can reflect the mechanical properties of the particles at the meso level and is the mesolevel manifestation of the macromechanical mechanisms at the particle level. The macromechanical mechanisms of geotechnical materials can be controlled by assigning and modifying the mesolevel contact parameters of the particles. According to previous simulation experience and by considering the existence of irregular coarse particles, the rrlinear contact model was selected as the contact model between ball-ball and ball-clump. The internal bending moment of the contact model increases linearly with the relative rotation accumulated at the contact point, with the rolling effect of regular spheres considered, which is usually used for contact analysis where the rotation effect between particle systems is very obvious. The linear contact model was selected as the contact model between the particles and the walls. The classification of the contact models between the particles of the numerical specimen is shown in Figure 5.

3.2.4. Calibration of Mesolevel Parameters. The calibration of mesoparameters is the most important and most difficult step of discrete element simulation because it is unlikely at present to establish the calculation formula between the macromechanical parameters of geotechnical materials and the mesomechanical parameters of particles. Therefore, in accordance with the usual practice of particle discrete elements, this study adopted the "trial and error method" and selected the test curve of slip zone soil whose coarse grain content is $50 \%$ in the medium-sized shear test as the calibration curve of the discrete element simulation. The fitting curve shown in Figure 6 was obtained through the continuous modification of various mesolevel parameters in the discrete element numerical model and a large number of simulation operations. The initial shear modulus and peak strength of the discrete element numerical test and test curve were close to each other, but the shear stress of the numerical specimen decreased near the completion of shear. However, the two are congruous in general, which indicates that the calibrated parameters and the established DEM numerical model are reliable. The specific values of the obtained parameters are shown in Table 2.

\section{DEM Simulation Results and Analysis}

4.1. Analysis of Dilatancy of Particles with Different Psephicity. Conventional shear test has limited capacity to observe the dilatancy and deformation characteristics of the specimen, but the dilatancy of the numerical specimen can be well monitored and recorded through the discrete element numerical simulation. In this study, the dilatancy of numerical rock samples with two different roundness under three different normal pressures $(50,100$, and $150 \mathrm{kPa})$ was recorded and derived by programming monitoring as shown in Figure 7. The vertical displacement produced by the numerical specimen in the shearing process was used as a measure of the dilatancy of the numerical specimen under different normal stresses and psephicity. A comparison revealed that under the same normal pressure, the dilatancy of the subround block model with better psephicity was less than the that of the angular blocks with poor psephicity, which is consistent with the dilatancy characteristics under macroscopic mechanics. At the mesolevel, worse particle psephicity leads to specimen with sharper angles and edges and more obvious dilatancy. In addition, the figure shows that in both models, the dilatancy decreases with the increase in normal stress. From the mesolevel perspective, this result suggests that the numerical specimens of slip zone soil are more prone to rotation and translation under low stress, and the model is more difficult to deflect because the limitation of pressure under higher stress.

4.2. Displacement Field Analysis of Numerical Specimens of Rock Blocks of Different Psephicity. Displacement-related changes in each particle of the numerical specimen can be accurately tracked and recorded in the PFC3D. These displacement changes can be visually and clearly expressed and analyzed by the displacement vector field. The arrow in the displacement field indicates the direction of particle displacement. The arrow body represents the size of the particle displacement. In an attempt to clearly counterpoint the changes in each particle in the numerical specimen's shear box during the shearing process, the arrow is given colors ranging from blue to red. The brighter the color, the greater the displacement.

Figures 8(a) and 8(b), respectively, show the displacement fields of rocks with sharp angle model (RSAM) and the subround block stone model (SBSM) after shearing. Observing the displacement fields of the two numerical specimens, we found that the displacement fields of the two models have "circumventing rocks" in the shearing process because of the existence of rocks in the specimens, that is, when the small particles in the shear box encounter large rocks, they will be squeezed by the big rocks and make a detour, and the phenomena of circumvention and dilatancy were more obvious in places where rocks were greater in number and more concentrated.

Figure 9 illustrates the changes in the displacement vector field of RSAM and SBSM under 100 and $150 \mathrm{kPa}$ during the shearing process. Comparative analysis revealed that the two numerical specimens with distinct psephicity have similarities and differences at different shearing stages. The similarity between the two is that in the consolidation phase before shearing, the displacement field of the discrete element numerical specimen is distributed in a scattered manner without obvious patterns, and after the shearing 


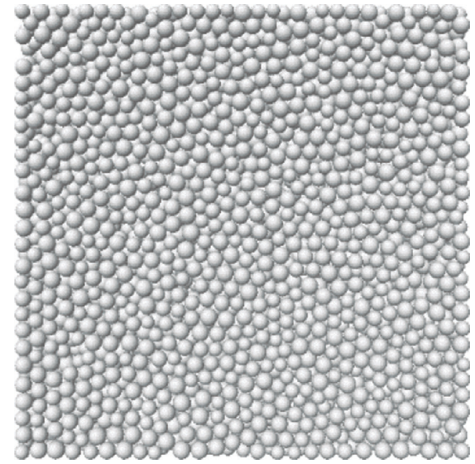

(a)

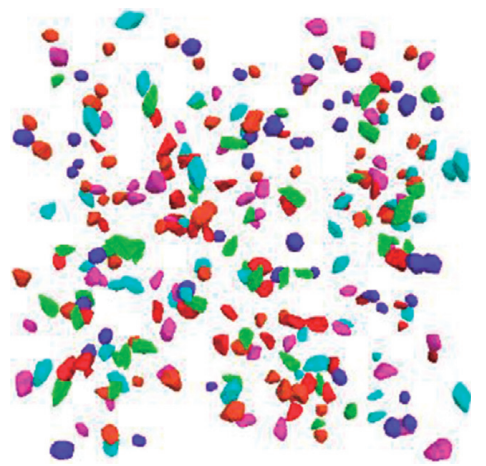

(b)

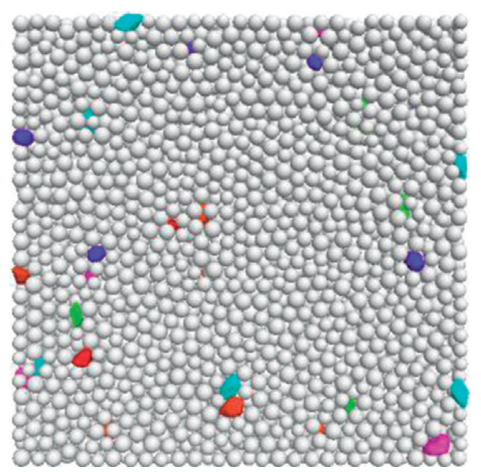

(c)

FIgUre 4: DEM numerical sample model of coarse and fine particle mixing. (a) Pure fine-grained soil. (b) Stone particle clump. (c) Coarse and fine particle mixing.

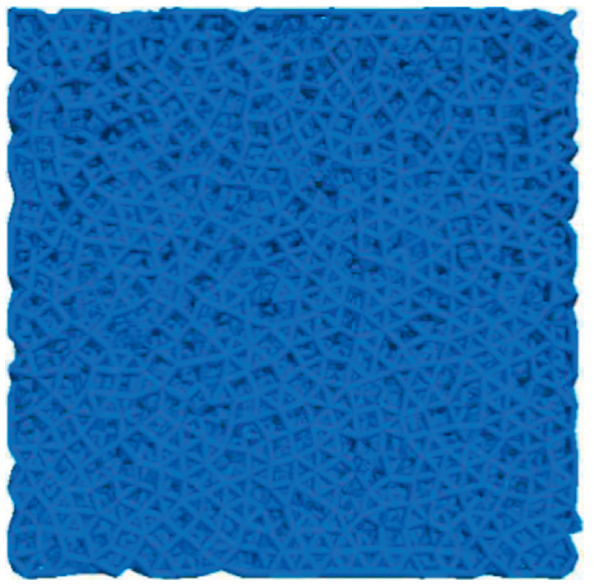

(a)

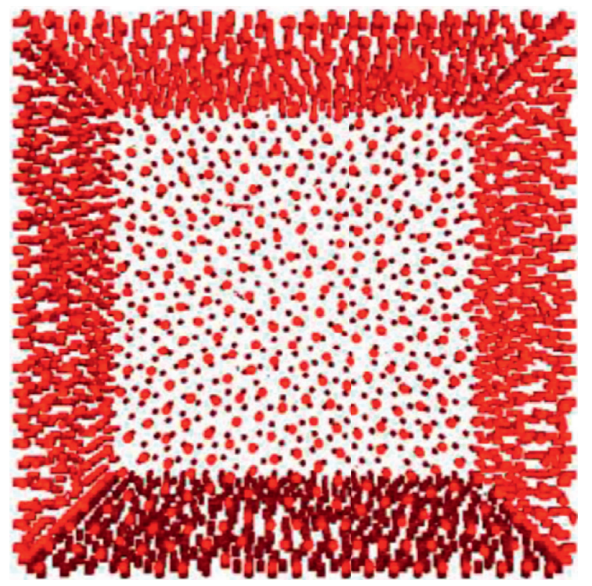

(b)

Figure 5: Mesoscopic contact model of numerical sample. (a) Contact between ball-ball and ball-pebble. (b) Contact between ball-facet.

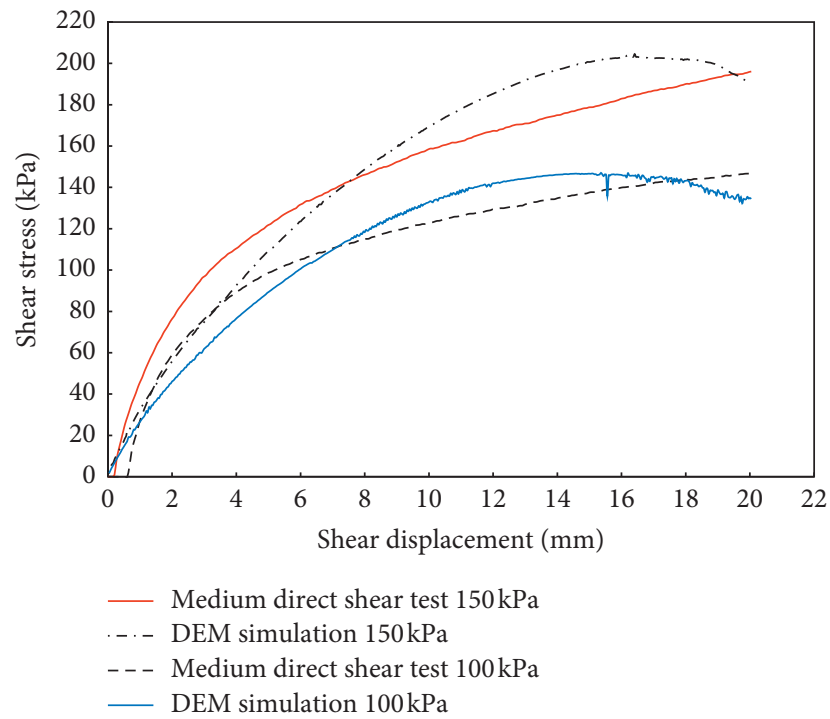

FIGURE 6: Fitting curves of shear test and discrete element numerical test.
TABLE 2: mesoscopic parameters of DEM contact model.

\begin{tabular}{lc}
\hline Mesostructure parameter & Value \\
\hline Density of fine grain soil particle/(kg/m $\left.\mathrm{m}^{3}\right)$ & 2000 \\
Effective modulus of fine granular soil $/(\mathrm{Pa})$ & $3.5 \times 10^{6}$ \\
The tangential stiffness ratio of fine granular soil (ball) & 1.2 \\
Friction coefficient of fine granular soil (ball) & 0.85 \\
Rolling friction coefficient of fine granular soil (ball) & 0.85 \\
Density of coarse particles $/\left(\mathrm{kg} / \mathrm{m}^{3}\right)$ & 2700 \\
Effective modulus of coarse particles $/(\mathrm{Pa})$ & $7.0 \times 10^{6}$ \\
Tangential stiffness ratio of coarse particle method & 1.5 \\
(clump) & 0.85 \\
Coarse particle friction coefficient (clump) & 0.85 \\
Coarse particle rolling friction coefficient (clump) & $3.5 \times 10^{7}$ \\
Effective modulus of wall/(Pa) & 0 \\
Friction coefficient between particles and wall &
\end{tabular}

starts, the particles in the direct shear box are subjected to the horizontal shearing forces and start to move along the direction of shearing. In this study, the direction and form of shearing are consistent with the actual medium-sized shear 


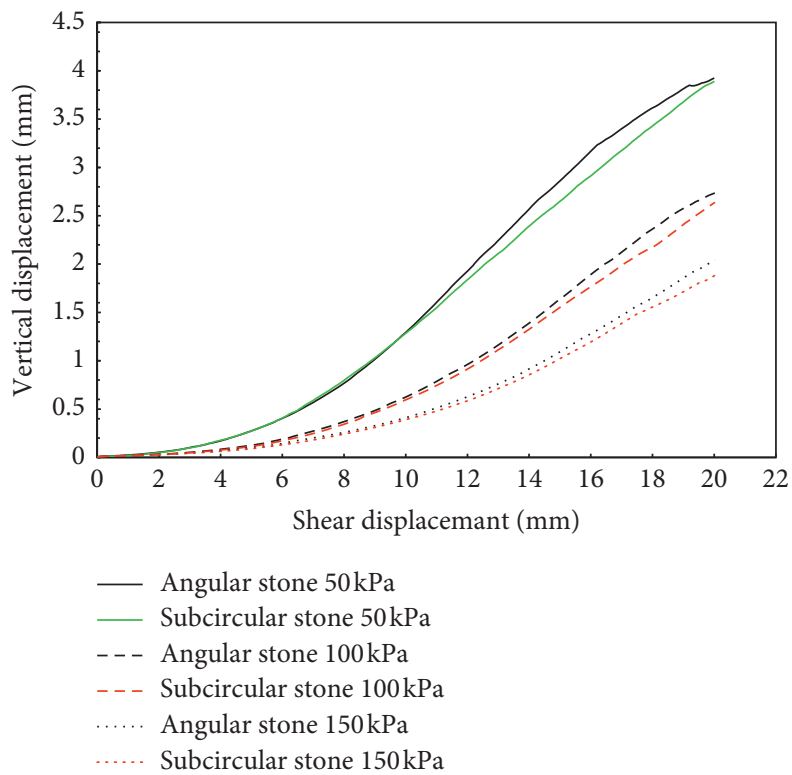

FiguRE 7: Dilatancy curves of rock models with different normal stresses and different roundness.

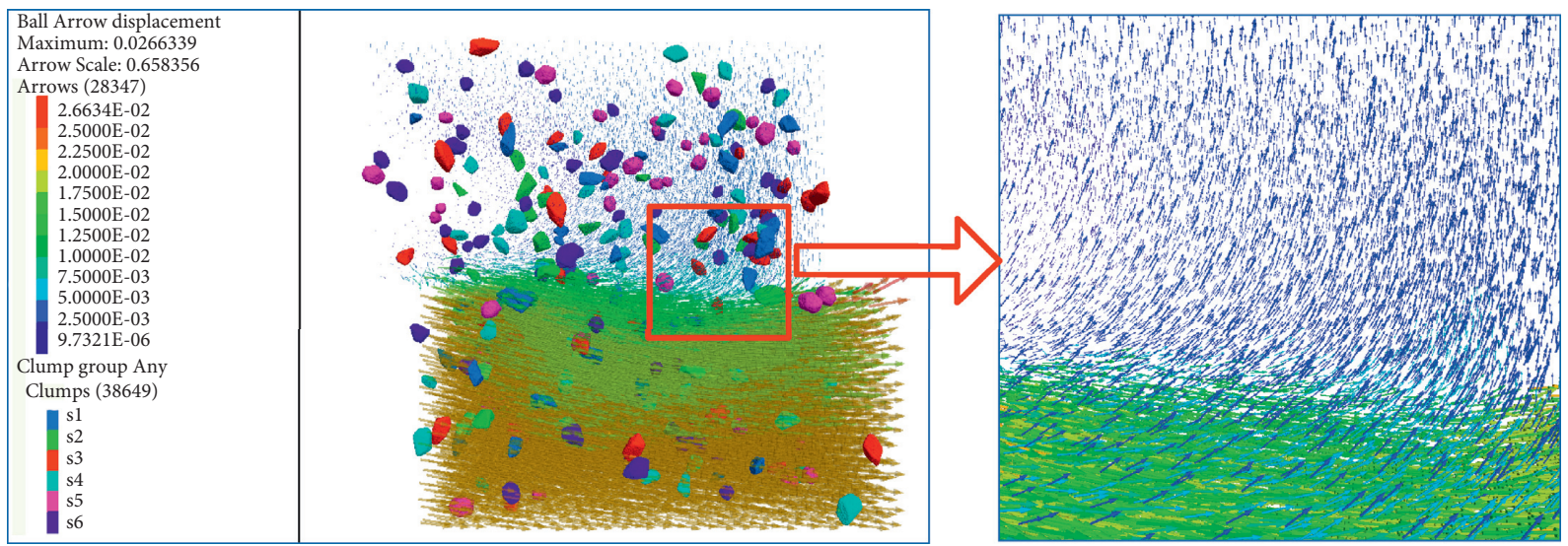

(a)

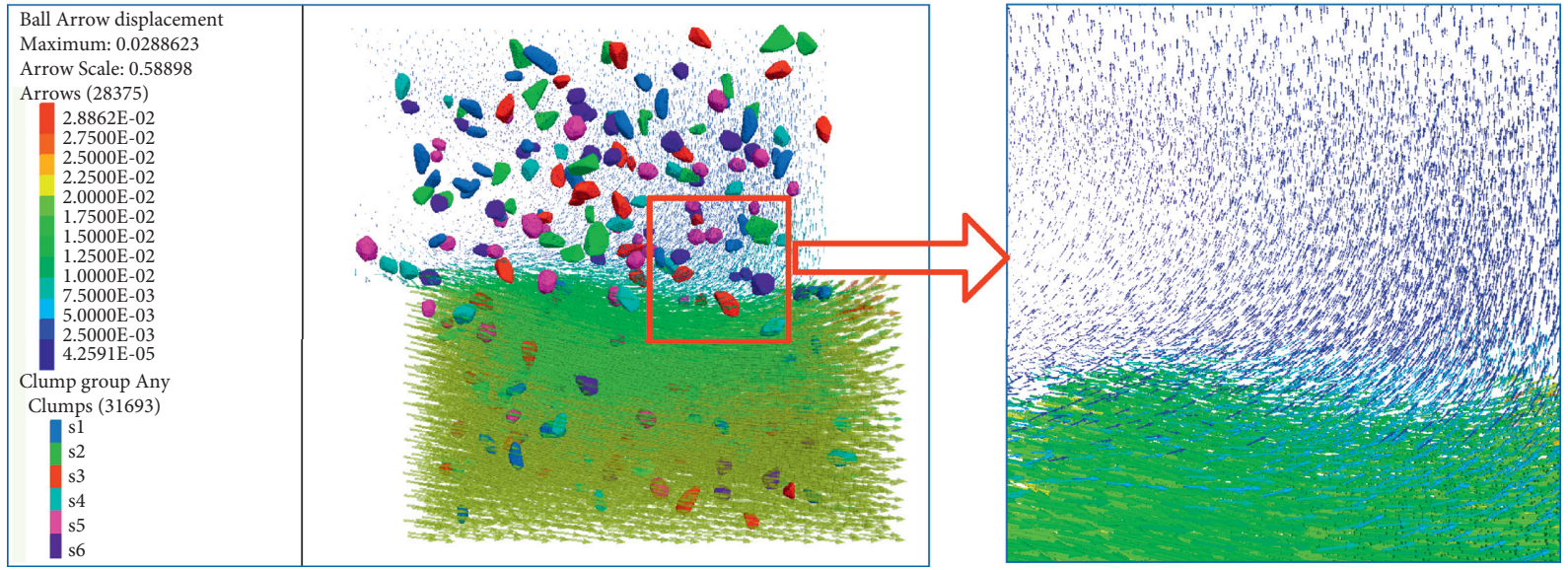

(b)

FIGURE 8: The phenomenon of rock around the displacement vector field of numerical sample. (a) RSAM displacement field. (b) SBSM displacement field. 


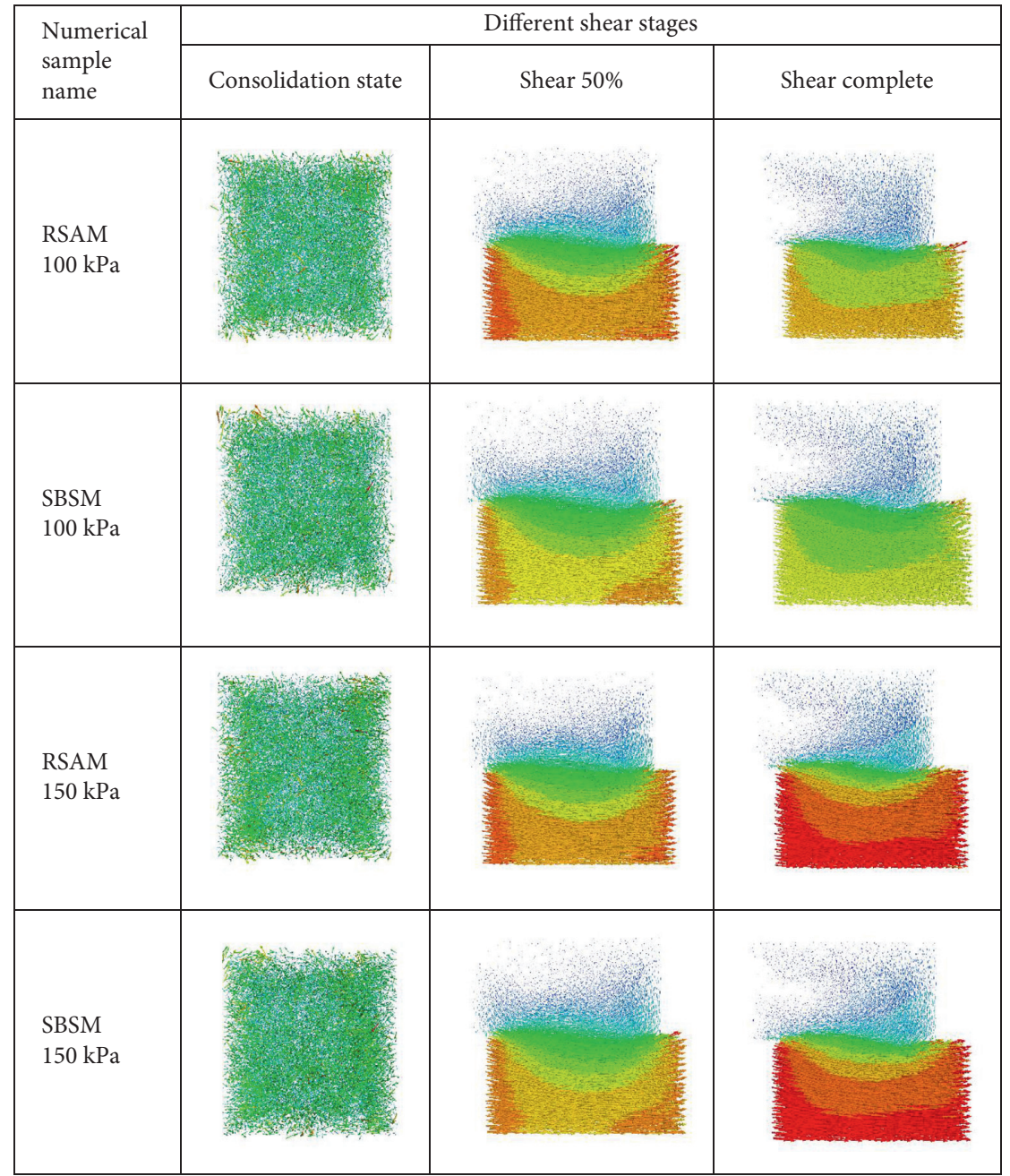

FIGURE 9: Variation of displacement field in the shear process of two kinds of numerical samples with different roundness of grinding.

tests: the upper shear box remained stationary, and the lower shear box was the active one. The lower box is the first to move and initiate the shear; therefore, the particles in the lower shear box are the first to display displacement, and the values (using the length and color of the arrow in the displacement field as the criterion) are much larger than those of the upper box. In addition, the similarity between the two is that the particle displacement and stratification in the lower box is obvious. The shear box can be divided into 3-4 parts from top to bottom with the middle of the shear box as the boundary. The delamination effect became more obvious as the normal pressure increased, and the shear displacement of the two numerical specimens increased with the increase in normal pressure. The displacement in the lower box was mostly horizontal or approximately so, but the displacement direction in the upper box had a larger deflection angle: from left to right, it exhibited an upward movement from $45^{\circ}$ to $90^{\circ}$, which reveals the reason for the obvious dilatancy of the numerical specimens from the mesolevel point of view.

The difference between the two models was because the displacement values in the box under RSAM that sheared at 100 and $150 \mathrm{kPa}$ were larger than those of SBSM. In addition, the upper part of the numerical specimen under the condition of sharp edges and corners was sheared. The distribution range of the particle displacement field in the box was slightly larger than that of the subround stone specimen, and the dilatancy was more obvious. This result shows that the shape of the particles will affect the movement process of the numerical specimen in shearing. A worse psephicity corresponds to a numerical specimen with sharper edges and corners and greater shear displacement.

\subsection{Comparative Analysis of Particle Contact Force Chains} under the Condition of Different Particle Shapes. 3D DEM was proven effective as an analysis method for the mesolevel contact force between particles. In bulk materials, the contact force between particles is transferred from one particle to adjacent ones. These contact forces can be visually displayed in the form of contact force chains in PFC. For instance, in Figure 10, the blue-colored chains are the contact force chains of the particles after the shearing was finished. Here, the particles became transparent to a certain degree. The rightward side of Figure 10 is the effect diagram after the force chains are enlarged. These contact force 


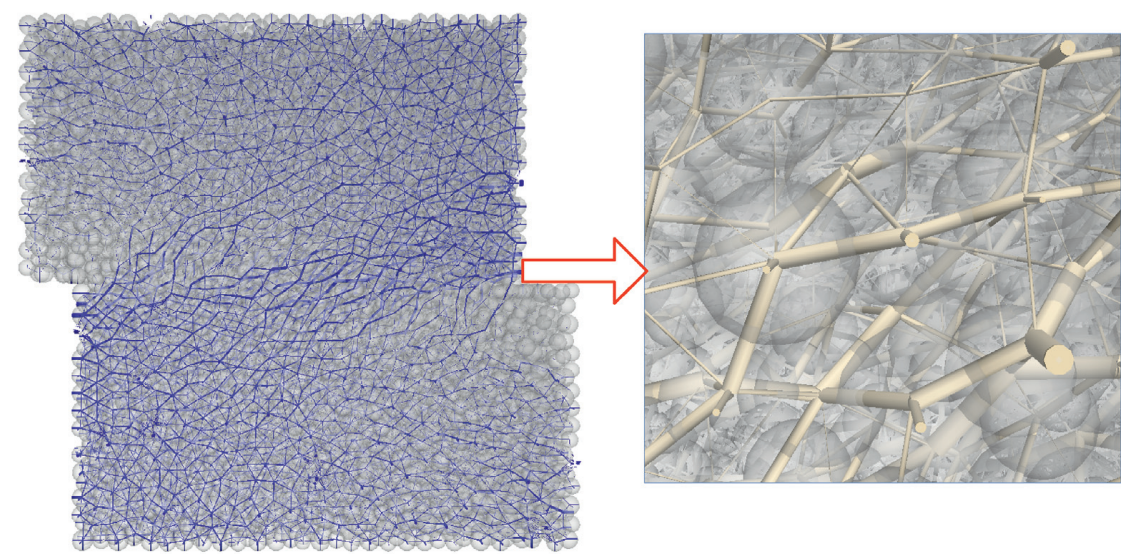

FIGURE 10: Schematic diagram of contact force chain.

chains can merge with each other, cross, and extend to form a complex force chain network. The contact network was classified between macroscopic units and microscopic particles in scale. It is the mesolevel embodiment of the macroscopic mechanical properties of granular materials, and its changing characteristics are congruous with the macroscopic stress-strain trend [40, 41].

This study statistically analyzed the contact numbers of two numerical specimens involving blocks with different psephicity in different shear stages under different normal pressures as shown in Table 3. The reduction ratio of the number of contacts of the numerical specimens before and after shear is defined as $\alpha=m_{2}-m_{1} / m_{1}$, where $m_{1}$ and $m_{2}$, respectively, represent the number of contacts in the numerical specimens in the consolidation phase before and after the completion of shearing. The comparative analysis shows that in the two numerical specimens, the number of contact force chains gradually reduced with the increase in shearing process because the contact or connection formed by the mutual contact between the numerical specimen particles is gradually destroyed under the action of horizontal shear stress. At $100 \mathrm{kPa}$ normal pressure, the " $\alpha$ " value of RSAM was $7.93 \%$, which was remarkably smaller than that of SBSM, but the ratio of contact number reduction is basically the same for both models at $150 \mathrm{kPa}$. In addition, the number of contacts at each stage before and after shearing was higher at $150 \mathrm{kPa}$ for RSAM and SBSM compared with that at $100 \mathrm{kPa}$, and the $\alpha$ value for both models dwindled at $150 \mathrm{kPa}$ compared with that at $100 \mathrm{kPa}$. This result can be interpreted from the mesolevel perspective and indicates that the particles of the numerical specimens are in closer contact with each other as the normal stress increases, which in turn gives rise to an increase in the number of contacts and a decline in the ratio of the decrease in the number of contacts before and after shear.

Figure 11 shows the evolution of the particle contact force chains of the two block models with different psephicity during shearing under $100 \mathrm{kPa}$ normal stress. The figure clearly presents that RSAM numerical specimen has a closer contact and thicker contact force chains and is more robust compared with SBSM. In addition, the contact force chains of both models are concentrated in the lower left, upper right, and middle areas of the shear box with the increase in shear displacement. The contact force chains between the upper and lower shear boxes are interconnected and interlaced to form more stable and thicker contact force chains, which intuitively show the changes and development trend of stress transfer between the particles of the numerical specimen in shear from the mesolevel point of view.

4.4. Analysis of Coordination Number. The magnitude of interfacial friction between the particles of a DEM numerical model is directly impinged by the effective contact area, and coordination number is the parameter used to measure the contact area and contact density between particles. Average coordination number $(\mathrm{ACN})$ is an important parameter in discrete elements used to evaluate whether a model has good and close contact. ACN can be calculated by the following equation: $\mathrm{ACN}=2 N_{p} / N_{a}$, where $N_{p}$ and $N_{a}$ represent the actual number of contacts and the total number of contacts in the discrete element model, respectively. The coordination number can be interpreted as the average number of contacts per particle in the numerical specimen, which can reveal the stress level and denseness of the specimen in shear from the mesolevel perspective. This study monitored the changes in porosity and coordination number during the shearing process by writing a program to set a measuring ball in the middle of the shear box of the numerical specimen to unearth the effect of rock blocks with different psephicity on the contact density between particles. The monitoring results are shown in Figure 12.

The figure presents that the development trends of the coordination number of the particles of the two numerical specimens with different psephicity are identical and can be divided into three phases, namely, the growth period, decline period, and stable period. First, the coordination number slightly increased with the increase in shear displacement, then decreased rapidly, and remained stable towards the end of shear. The contact density of the two numerical specimens also increased initially and then decreased. The analysis was unfolded from the mesolevel perspective because the numerical specimens were squeezed and filled with fine particles in the pores under the action of shear stress, which 
TABLE 3: Contact number in different shear stages.

\begin{tabular}{lccc}
\hline \multirow{2}{*}{ Numerical sample name } & \multicolumn{2}{c}{ Shear stages } & \\
& Consolidation state & Shear 50\% & Shear complete \\
\hline RSAM $(100 \mathrm{kPa})$ & 135,892 & 130,636 & 125,111 \\
RSAM $(150 \mathrm{kPa})$ & 146,072 & 143,280 & 137,364 \\
SBSM $(100 \mathrm{kPa})$ & 132,903 & 127,684 & 121,405 \\
SBSM $(150 \mathrm{kPa})$ & 142,523 & 139,947 & 5.93 \\
\hline
\end{tabular}

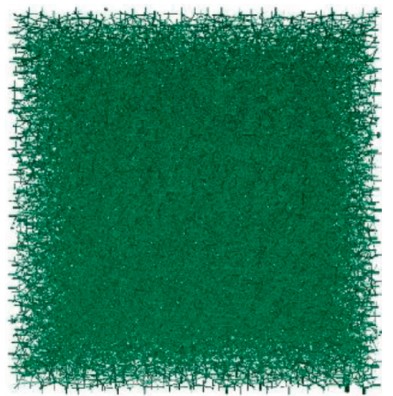

(a)

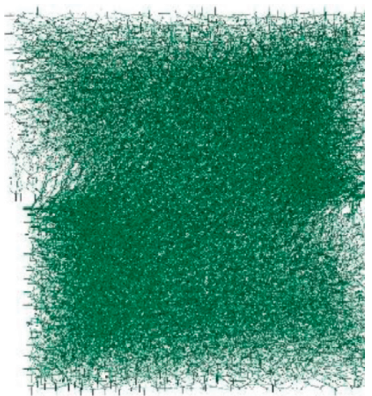

(b)

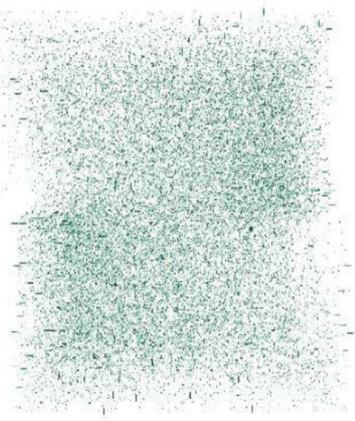

(e)

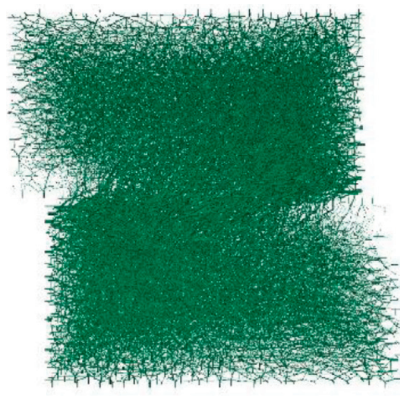

(c)

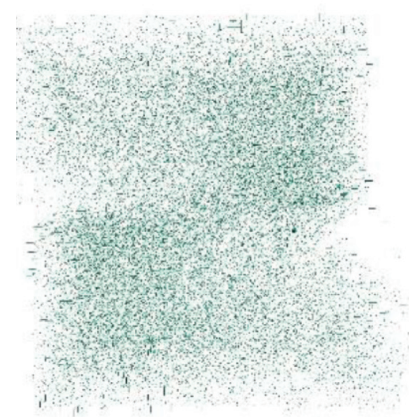

(f)

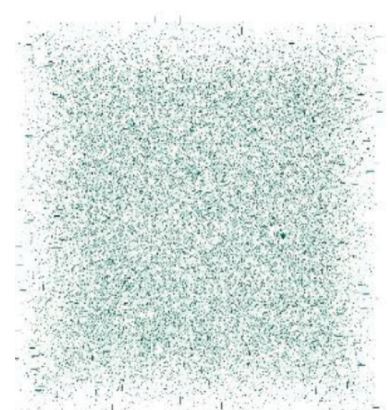

(d)

FIGURE 11: Contact force chain evolution of stone numerical samples with different roundness in the shear process under $100 \mathrm{kPa}$ normal stress. (a) RSAM consolidation state. (b) RSAM shear 50\%. (c) RSAM shear complete. (d) SBSM consolidation state. (e) SBSM shear 50\%. (f) SBSM shear complete.

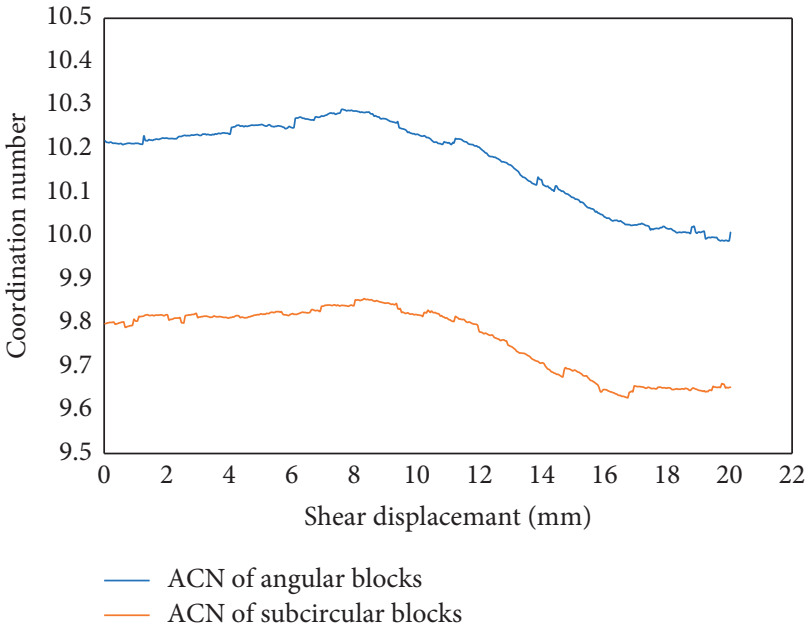

Figure 12: Coordination number comparison of two numerical samples with different grinding roundness at $150 \mathrm{kPa}$. made the specimens contact more closely, and then with the increase in shear displacement, the left side of the upper shear box and the right side of the lower shear box are exposed, and the contact or connection between the particles is are damaged, which makes the contact density decrease rapidly. Furthermore, RSAM had a coordination number with larger initial value and longer growth period than SBSM, which indicates that particle shape can effectively affect the contact density of the numerical specimens, and the model involving blocks of sharper angles and poorer psephicity had better contact density and larger coordination number.

\section{Discussion}

On the basis of the 3D scanning of the test rocks, refined 3D DEM numerical shear tests were performed to initially investigate the effect of rock particles with different degrees of 
roundness on the macrolevel and mesolevel mechanical properties of slip zone soils. Wang and Wang, and Yang and Li $[42,43]$ only used regular spheres and simple combinations of spheres to simulate soil particles in 3D discrete element numerical shear tests, which differ considerably from the unevenness and irregularity of particles in actual soil. In comparison, the irregular coarse-grained numerical specimens used in this study are closer to the mechanical properties of actual soil. In addition, although Zheng et al. [44] also conducted 3D scanning and discrete element numerical analysis of coarse particles, such as rocks, they imported only one 3D scanned rock when generating the discrete element numerical specimens, which caused a shortage on the credibility of its research conclusions. In comparison, the number of coarse particles imported and generated in the present article is closer to that of real specimens, and the research conclusions obtained are more credible. In addition, because of the limitations of current laboratory apparatus, the shearing-related apparatus used in this study to conduct the slip zone soil test is not yet able to conduct shear tests under high-level normal pressure. Therefore, three shear tests were conducted under the normal pressures of 50,100 , and $150 \mathrm{kPa}$, and shear tests under higher normal pressure and discrete element simulation studies are expected to be conducted after the test conditions are improved. The indoor tests and discrete element numerical analysis also need to be consummated.

\section{Conclusions}

In this study, two numerical specimen models of slip zone soil with rock blocks of different psephicity were created through 3D scanning technique based on 3D DEM, and the macroscopic and mesolevel mechanical and deformation damage characteristics of both models under direct shear loading were investigated. According to the present simulation study, the following conclusions can be drawn.

(1) Through the 3D scanning technology, a three-dimensional numerical model of the test blocks can be accurately constructed and combined with the discrete element method, it can effectively analyze the micromechanical properties of the slip zone soil; the selected resistance linear contact model can be used for the discrete element simulation of slip zone soil containing coarse particles.

(2) The psephicity and particle shape of the blocks impose an important influence on the dilatancy of the slip zone soil. The worse the psephicity and the more irregular the particle shape, the more significant the dilatancy of the numerical specimen.

(3) The psephicity and particle shape of the blocks have a remarkable effect on the micromechanical characteristics of the slip zone soil. The block model characterized by irregular particle shape and sharp angles tends towards greater displacement of particles, wider distribution of displacement field, as well as more significant delamination effect.
(4) The block model with better psephicity has fewer contact force chains than the model with poor psephicity, and its contact force chains are relatively fine and sparse, not as thick and stable as the contact force chain network formed by the latter, and the proportion of its contact force chains reduced after shearing is also greater.

(5) In the whole course of shearing, the numerical specimen of the slip zone soil composed of rock blocks of poorer psephicity exhibits a greater coordination number than that of the model featured by better psephicity, and the internal contact of the former model is closer.

\section{Data Availability}

All the data supporting this study are included within the article.

\section{Conflicts of Interest}

The authors declare no conflicts of interest.

\section{Acknowledgments}

This research was supported by Heilongjiang Provincial Natural Science Foundation of China (Grant no. LH2019D001) and China Postdoctoral Science Foundation (Grant no. 2018M631895).

\section{References}

[1] X. L. Liu, J. H. Deng, and G. T. Li, "Shear strength properties of slip soils of landslides: an overview," Rock and Soil Mechanics, vol. 25, no. 11, pp. 1849-1854, 2004.

[2] A. W. Skempton, "Residual strength of clays in landslides, folded strata and the laboratory," Géotechnique, vol. 35, no. 1, pp. 3-18, 1985.

[3] S. Gibo, K. Egashira, and M. Ohtsubo, "Residual strength of smectite-dominated soils from the Kamenose landslide in Japan," Canadian Geotechnical Journal, vol. 24, no. 3, pp. 456-462, 1987.

[4] H. Shuzui, "Process of slip-surface development and formation of slip-surface clay in landslides in tertiary volcanic rocks, Japan," Engineering Geology, vol. 61, no. 4, pp. 199-220, 2001.

[5] B. Tiwari and H. Marui, "Objective oriented multistage ring shear test for shear strength of landslide soil," Journal of Geotechnical and Geoenvironmental Engineering, vol. 130, no. 2, pp. 217-222, 2004.

[6] B. P. Wen, A. Aydin, N. S. Duzgoren-aydin, Y. R. Li, H. Y. Chen, and S. D. Xiao, "Residual strength of slip zones of large landslides in the three Gorges area, China," Engineering Geology, vol. 93, no. 3-4, pp. 82-98, 2007.

[7] X. P. Chen and D. Liu, "Residual strength of slip zone soils," Landslides, vol. 11, no. 2, pp. 305-314, 2014.

[8] X. P. Chen, J. W. Huang, S. H. Yin, and J.-z. Zheng, "Experimental study of strength property of slip zone soils," Rock and Soil Mechanics, vol. 32, no. 11, pp. 3212-3218, 2011.

[9] D. Liu and X. P. Chen, "Microscopic observation and analysis of ring shear surface of slip zone soil," Chinese Journal of Rock 
Mechanics and Engineering, vol. 32, no. 9, pp. 1827-1834, 2013.

[10] D. Liu and X. P. Chen, "Laboratory test and parameter back analysis of residual strength of slip zone soils," Journal of South China University of Technology, vol. 42, no. 2, pp. 81-87, 2014.

[11] Y. R. Li, B. P. Wen, A. Aydin, and N. P. Ju, "Ring shear tests on slip zone soils of three giant landslides in the three Gorges project area," Engineering Geology, vol. 154, pp. 106-115, 2013.

[12] Z. Zou, Q. Zhang, C. Xiong et al., "In situ shear test for revealing the mechanical properties of the gravelly slip zone soil," Sensors, vol. 20, no. 22, pp. 6531-6547, 2020.

[13] S. S. Ren, Y. S. Zhang, N. X. Xu, and R. Wu, "Mesoscopic response mechanism of shear surface roughness and residual," Chinese Journal of Geotechnical Engineering, vol. 43, no. 8, pp. 1473-1482, 2021.

[14] L. Q. Wu, M. Liu, and J. Zhang, "Experimental study on shear characteristics of gravelly slip soil," Pearl River, vol. 40, no. 3, pp. 23-28, 2019.

[15] B.-P. Wen and X.-Z. Jiang, "Effect of gravel content on creep behavior of clayey soil at residual state: implication for its role in slow-moving landslides," Landslides, vol. 14, no. 2, pp. 559-576, 2017.

[16] S. S. Ren, Y. S. Zhang, N. X. Xu, R. Wu, X. Liu, and G. Du, "Mobilized strength of sliding zone soils with gravels in reactivated landslides," Rock and Soil Mechanics, vol. 42, no. 3, pp. 863-873+881, 2021.

[17] J. W. Jiang, W. Xiang, and X. Y. Zhang, "Research on mechanical parameters of intact sliding zone soils of Huangtupo landslide based on CT scanning and simulation tests," Chinese Journal of Rock Mechanics and Engineering, vol. 30, no. 5, pp. 1025-1033, 2011.

[18] X. L. Chang, G. Ma, W. Zhou, and C. Zhou, "Influences of particle shape and inter-particle friction angle on macroscopic response of rockfill," Chinese Journal of Geotechnical Engineering, vol. 34, no. 4, pp. 646-653, 2012.

[19] Y. Luo, X. N. Gong, and F. Lian, "Simulation of mechanical behaviors of granular materials by three-dimensional discrete element method based on particle flow code," Chinese Journal of Geotechnical Engineering, vol. 30, no. 2, pp. 292-297, 2008.

[20] J. Zhou and Y. Chi, "Simulating soil properties by particle flow code," Acta Mechanica Solida Sinica, vol. 25, no. 4, pp. 377382, 2004

[21] D. O. Potyondy and P. A. Cundall, "A bonded-particle model for rock," International Journal of Rock Mechanics and Mining Sciences, vol. 41, no. 8, pp. 1329-1364, 2004.

[22] H. T. Liu and X. H. Cheng, "Discrete element analysis for size effects of coarse-grained soils," Rock and Soil Mechanics, vol. 30, no. S1, pp. 287-292, 2009.

[23] X. L. Ding, Y. X. Li, and X. Wang, "Particle flow modeling mechanical properties of soil and rock mixtures based on digital image," Chinese Journal of Rock Mechanics and Engineering, vol. 29, no. 3, pp. 477-484, 2010.

[24] M. Jiang, T. Jiang, G. B. Crosta, Z. Shi, H. Chen, and N. Zhang, "Modeling failure of jointed rock slope with two main joint sets using a novel DEM bond contact model," Engineering Geology, vol. 193, pp. 79-96, 2015.

[25] Q. Zhang, X. G. Wang, Y. F. Zhao, and J. W. Zhou, "Discrete element simulation of large-scale triaxial tests on soil-rock mixtures based on flexible loading of confining pressure," Chinese Journal of Geotechnical Engineering, vol. 41, no. 8, pp. 1545-1554, 2019.
[26] M. Jiang, A. Zhang, and Z. Shen, "Granular soils: from DEM simulation to constitutive modeling," Acta Geotechnica, vol. 15, no. 7, pp. 1723-1744, 2020.

[27] X. M. Jia, H. J. Chai, and Y. R. Zheng, "Mesomechanics research of large direct shear test on soil and rock aggregate mixture with particle flow code simulation," Rock and Soil Mechanics, vol. 31, no. 9, pp. 2695-2703, 2010.

[28] W. Q. Huang and Y. Lu, "3D DEM simulation of random packing of particulates under gravity," Chinese Journal of Geotechnical Engineering, vol. 28, no. 12, pp. 2139-2143, 2006.

[29] Y. Yan, J. F. Zhao, and S. Y. Ji, "Discrete element analysis of the influence of rock content and rock spatial distribution on shear strength of rock-soil mixtures," Engineering Mechanics, vol. 34, no. 6, pp. 146-156, 2017.

[30] Y. Liu, S. C. Ching, D. Zhang, and C. L. Wang, "Analytical solution of stress-fabric relationship and failure of granular materials in three dimensions," Chinese Journal of Geotechnical Engineering, vol. 36, no. 3, pp. 401-408, 2014.

[31] Z. Li, Z. Liu, P. Cheng, and Z. Li, "Micro-characteristics and meso-shear mechanism of the soils of a slip zone in a landslide," Mechanics of Advanced Materials and Structures, pp. 1-12, 2021

[32] L. Kong and R. Peng, "Particle flow simulation of influence of particle shape on mechanical properties of quasi-sands," Chinese Journal of Rock Mechanics and Engineering, vol. 30, no. 10, pp. 2112-2119, 2011.

[33] C. G. Zhang, Z. Y. Yin, Z. X. Wu, and Y. F. Jin, "Three-dimensional discrete element simulation of influence of particle shape on granular column collapse," Rock and Soil Mechanics, vol. 40, no. 3, pp. 1197-1203, 2019.

[34] W. J. Xu and S. Wang, "Meso-mechanics of soil-rock mixture with real shape of rock blocks based on 3D numerical direct shear test," Chinese Journal of Rock Mechanics and Engineering, vol. 35, no. 10, pp. 2152-2160, 2016.

[35] L. Jin and Y. W. Zeng, "Refined simulation for macro-and meso-mechanical properties and failure mechanism of soilrock mixture by 3D DEM," Chinese Journal of Rock Mechanics and Engineering, vol. 37, no. 6, pp. 1540-1550, 2018.

[36] P. C. Wang and J. K. Liu, "Effect of particle shape on the shear behavior of open-graded crushed aggregate," Rock and Soil Mechanics, vol. 38, no. 8, pp. 2198-2202, 2017.

[37] S. W. Zhao, X. W. Zhou, W. H. Liu, and P. Liu, "DEM simulation of direct shear tests considering particle angularity effect," Rock and Soil Mechanics, vol. 36, no. 1, pp. 602-608, 2015.

[38] Z. P. Zhang, Q. Sheng, X. D. Fu, H. X. Luo, and L. Z. Dan, "Research on numerical direct shear test of soil-rock mixture based on particle flow code simulation," Journal of Basic Science and Engineering, vol. 29, no. 1, pp. 135-146, 2021.

[39] S. Yang and X. Q. Li, "Simulation analysis of large direct shear test of soil and rock mixture based on PFC3D," Advanced Engineering Sciences, vol. 52, no. 3, pp. 78-85, 2020.

[40] L. Zhang, N. G. Nguyen, S. Lambert, F. Nicot, F. Prunier, and I. D. Maigre, "The role of force chains in granular materials: from statics to dynamics," European Journal of Environmental and Civil Engineering, vol. 21, no. 7-8, pp. 874-895, 2017.

[41] R. G. Wan and P. J. Guo, "Effect of microstructure on undrained behaviour of sands," Canadian Geotechnical Journal, vol. 38, no. 1, pp. 16-28, 2001.

[42] X. Wang, Z. Wang, X. Jing, and C. Xiao, "A macro-micro study and distinct element simulation on large-scale shear test of coarse-grained soil," Journal of Shenzhen University Science and Engineering, vol. 37, no. 3, pp. 279-286, 2020. 
[43] S. Yang and X. Q. Li, "Shear simulation and macro meso analysis of sand based on the PFC3D," Chinese Journal of Computational Mechanics, vol. 36, no. 6, pp. 777-783, 2019.

[44] B. N. Zheng, D. Y. Ding, D. Zhang, L. Gao, and J. Yang, "CT scanning and PFC modeling combined 3D method for gravelbearing slip soil," Journal of Engineering Geology, vol. 27, no. 3, pp. 569-576, 2019. 